\title{
Real-time Medical Visualization of Human Head and Neck Anatomy and its Applications for Dental Training and Simulation
}

\author{
Paul Anderson ${ }^{1}$, Paul Chapman, Minhua Ma, and Paul Rea ${ }^{2}$ \\ ${ }^{1}$ Digital Design Studio, Glasgow School of Art, \\ The Hub, Pacific Quay, Glasgow, G51 1EA, UK \\ \{p.anderson, p.chapman,m.ma\}@gsa.ac.uk \\ Phone: +44 (0)141 566-1478 \\ ${ }^{2}$ Laboratory of Human Anatomy, School of Life Sciences, \\ College of Medical, Veterinary and Life Sciences, University of Glasgow, G12 8QQ, UK \\ Paul.Rea@glasgow.ac.uk \\ Phone +44(0)141 330-4366
}

Running title: Real-time Visualization of Head and Neck Anatomy

\begin{abstract}
The Digital Design Studio and NHS Education Scotland have developed ultra-high definition real-time interactive 3D anatomy of the head and neck for dental teaching, training and simulation purposes. In this paper we present an established workflow using state-of-the-art 3D laser scanning technology and software for design and construction of medical data and describe the workflow practices and protocols in the head and neck anatomy project. Anatomical data was acquired through topographical laser scanning of a destructively dissected cadaver. Each stage of model development was clinically validated to produce a normalised human dataset which was transformed into a real-time environment capable of large-scale 3D stereoscopic display in medical teaching labs across Scotland, whilst also supporting single users with laptops and PC. Specific functionality supported within the 3D Head and Neck viewer includes anatomical labelling, guillotine tools and selection tools to expand specific local regions of anatomy. The software environment allows thorough and meaningful investigation to take place of all major and minor anatomical structures and systems whilst providing the user with the means to record sessions and individual scenes for learning and training purposes. The model and software have also been adapted to permit interactive haptic simulation of the injection of a local anaesthetic.
\end{abstract}

Keywords: dental simulation, haptic interaction, head and neck anatomy, laser scanning, medical visualization, real-time simulation, real-time visualization

\section{Background}

3D scanning technology, including laser scanning and white light scanning, are being used to explore applications in medicine already routinely used in other fields. In healthcare, the technology has also been used in development of prostheses as translated scan data is immediately usable in computer aided design software, improving the speed of development of the prosthesis. One of the limitations of laser scanning technology is that it is only able to capture and reconstruct the outer surface of the body [1], therefore the scans do not have any internal structure and physical properties regarding skeleton, skin or soft tissues of the scanned human body, unless it is combined with cadaveric dissection [2].

On the other hand, medical visualization based on direct and indirect volumetric visualization uses data derived from 3D imaging modalities such as CT, MRI, cryosection images, or confocal microscopy. Although, the visualization is generally accurate, it only represents a particular human body or cadaveric specimen. Demonstrating a normalised anatomically correct model is difficult due to the source of data-the largely elderly population of cadaveric specimens. In indirect volume visualization where individual surface models are reconstructed, mistakes and inaccuracy might be introduced from the manual or automatic segmentation

\footnotetext{
${ }^{1}$ Corresponding author
} 
process; whereas in direct volumetric visualization, interactivity is limited since surface geometries are not reconstructed. As a result, the users are not able to manipulate the model (volumetric data) as they could on surface models. Functions such as virtual dissection, e.g. disassemble/reassemble, and studying individual substructures are not possible. Furthermore, each imaging modality has its limitations, for example, for cryosections, the cadaver had to be segmented into large blocks which results in a loss of data at certain intervals; for CT/MRI images, segmentation rarely focuses on very thin anatomic structures [3] such as fascia. Developing a model that can present thin anatomic structures would be of great interest to medical professionals and trainees. However, the ability to accurately segment very thin structures is a challenging and substantial task.

In this paper we present an established workflow using state-of-the-art laser scanning technology and software for design and construction of 3D medical data and describe the workflow practices and protocols in the Head and Neck Anatomy project at the Digital Design Studio (DDS). The workflow overcomes the above limitations in volumetric visualization and surface anatomy.

This work was conducted by a well-established, unique, multi-disciplinary team drawn from art, technology and science. The team includes computer scientists, 3D modellers and animators, mathematicians, artists and product designers, and champions a culture of research and creativity which is fast moving, highly productive, externally engaged and autonomous. This successful academic hybrid model is built upon strong collaborative partnerships directly with industry and end users, resulting in tangible real-world outputs. We have sought to establish a balanced portfolio of research, teaching and commercialisation operating within a state-of-the-art, custom built facility located within the heart of the Digital Media Quarter in Glasgow. The DDS houses one of the largest virtual reality and motion capture laboratories in the world ensuring that we are at the forefront of digital innovation, developing products focussed on simulated virtual environments, highly realistic digital 3D models and prototypes, user interfaces and avatars. The virtual reality laboratory is at a huge scale enabling 3040 users simultaneously to experience live real-time simulation and interaction as shown in Figure 1.

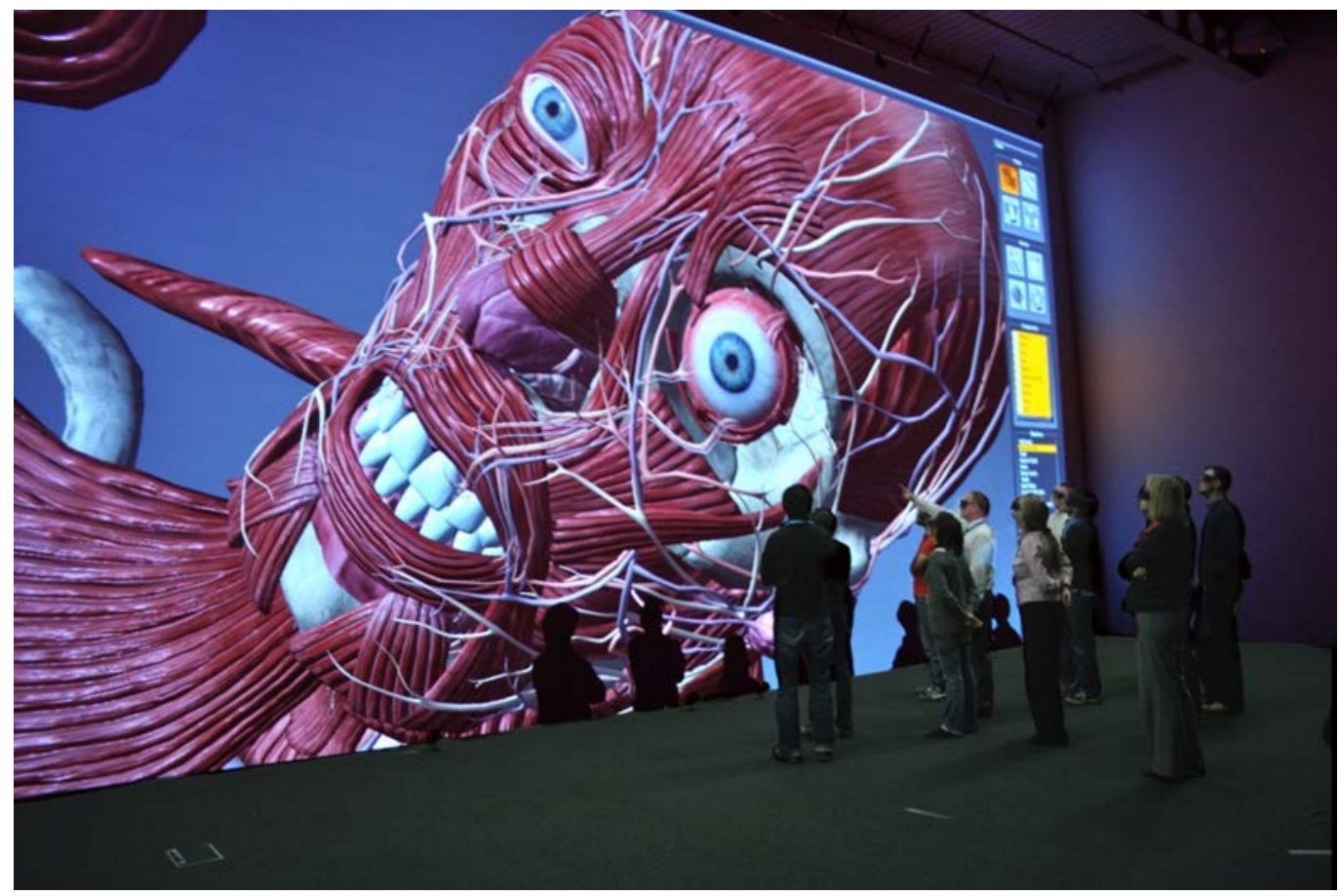

Figure 1. The Head and Neck system was presented in the large scale Virtual Reality laboratory

The work also involves the Scottish Medical Visualization Network, a collaborative initiative that has brought together 22 different medical disciplines and allied healthcare professionals across 44 organisations in Scotland to pursue excellence in medical visualization. Through this network, we created 3D digital models of selected anatomy that have been used to educate health professionals [4] and have also been used to support activities 
such as pre-operative planning, risk reduction, surgical simulation and increased patient safety. The work has also received significant recognition in recent major publications such as the RCUK report Big Ideas for the Future [5].

\section{Development of Head and Neck Anatomy}

NHS Education Scotland (NES) launched a European tender to develop a four strand work package to develop digital content for interactive Head and Neck anatomy, instrument decontamination, virtual patients and common disease processes for dentistry. This was a complex project requiring high levels of interaction across multidisciplinary development partners, in order to build digital anatomy (the 3D Definitive Head and Neck) and other digital products for medical teaching, across a distributed network of centres and primary care settings. The research took place within established protocols concerned with government legislation and patient interaction where the security of data and appropriate interface development were key factors.

This paper will discuss and focus on the Work Package A, i.e. the 3D interactive Head and Neck Anatomy. The aim of this project, commissioned by NES, was to complete the construction of the world's most accurate and detailed anatomical digital model of the head and neck using state-of-the-art data acquisition techniques combined with advanced, real-time 3D modelling skills and interactive visualization expertise. It was felt essential that this digital model must be capable of real-time interaction supporting both medical training and personal exploration, with all 3D data models and information being fully annotated, medically validated, and interactively "disassembled" to isolate and study individual substructures then reassembled at the touch of a button. In order to create a truly accurate interactive digital 3D model of head and neck anatomy it was essential to base the models upon real data acquired from both cadaveric and live human subjects. The full range of digital products was formally launched in April 2013. User feedback from NES medical teaching centres and primary care settings has been extremely positive.

This model integrates different tissue types, vasculature, and numerous substructures that are suitable for both the casual user and, in particular, those engaged in medical learning and teaching. Our model and software interface provides fluid and intuitive ways to visualise and encourage meaningful engagement with human anatomy amongst diverse audiences and is currently being well received by medical trainees, clinicians and the general public. Our 3D digital model development process, including data acquisition, model construction, interface design and implementation has been critically evaluated and validated by multi-disciplinary experts in the fields of medicine and computing science. Our extensive collaborative research network includes senior clinicians, surgical consultants, anatomists and biomedical scientists within the NHS, formal links with the medical schools within the Universities of Glasgow, Edinburgh, Dundee, Manchester and London, and other key specialists in the Scottish Medical Visualization Network.

\section{A Review of Anatomical Visualization Systems}

We continually monitor the medical visualization landscape for similar visualization products, and conduct regular exhaustive reviews of other digital datasets to ensure we maintain high levels of innovation and that our activities make a significant contribution to the field. Our development team, consisting of licensed anatomists, computer scientists and 3D modellers, has scrutinised all relevant competition worldwide. It is well known that there have been numerous datasets, models and atlases of human anatomy developed over the years. The first such major undertaking was the Visible Human which produced whole-body datasets based on male and female cadavers. The value and impact of this initial dataset were immediate: clinicians, scientists and educators throughout the world quickly accessed and downloaded this information, which became the de facto dataset on human anatomy for years (despite its limitations). The massive and strongly positive response to the introduction of the Visible Human demonstrates the need for the existence of, and accessibility to, anatomical information that is comprehensive, reliable and easy to use. Since then, several datasets have emerged that have attempted to contain either partial or whole-body anatomical information. 
There are approximately twelve anatomical visualization datasets available which vary widely in both quality and viability. We outline the functionality and fidelity of seven of them, below, in order to provide a comparison with our Head and Neck project.

VisibleBody [6] is a web-based, downloadable 3D human full body viewer. The dataset is proprietary and claimed as 'extremely accurate'. Tools include rotate, zoom, select single or multiple parts, labels, transparency, search and locate anatomical structures by name, cut and view internal structure of organs. Google Body Browser [7] is a basic interactive 3D human body viewer using Zygote Media Group's dataset. The software is free to use. BodyBrowser requires WebGL which requires latest versions of browsers. It features rotate, zoom, select single parts, labels on each part, transparency, and iteration between layers (skin, muscles, bones, organs, brain and nerves). Primal Pictures [8] produces various pieces of software that each visualise various parts of the body, e.g. one for the head and neck, another for shoulders and hands. The dataset is proprietary and also includes volumetric data from MRI, CT and cryosection images. It provides rotate, zoom, dynamic search, part selection, labels and extraction of metadata tools including extra educational information for each body part. Cyber Anatomy Med [9] is a medical visualization educational package with models constructed from CT/MRI volumetric datasets. It features rotation, translation, zoom, selection of parts, dissection mode, search, transparency, labels, culling planes that use MRI, CT and cryosection data, hide, reveal, explode, implode, screenshot capturing and stereoscopic 3D support. The Visible Human Project [10] is a complete, anatomically detailed, 3D representation of the normal male and female human. Acquisition of transverse CT, MR and cryosection images of representative male and female cadavers have been completed. Volumetric datasets are difficult to visualise due to the many complex steps required to process the data-it requires a significant level of user expertise, and the segmentation of the datasets is equally complex. 3D4Medical [11] produces medical visualization software for reference in health and fitness markets. Their latest applications are 'interactive' although the models are not truly interactive as they use pre-rendered animations that only allow the user to manipulate viewpoints in predetermined vertical or horizontal paths. This pre-rendered approach also results in a 'grainy' display when the user zooms into the 3D model. BodyViz [12] is a 3D medical imaging software that combines volumetric visualization with a game controller interface. Apart from basic navigation controls, it provides: clipping plane on arbitrary angles; Xbox controller; view different tissue types (e.g. bones, muscles); and customer created annotations. It can also visualise live patients' CT/MRI data.

We believe that the Head and Neck project improves on the above products in several ways.

- Accuracy/clinical value. Some of the described products above are oversimplified, lacking detail and reproducing anatomical errors due to a reliance on 2D sources such as Netter-type illustrations. Several omit relevant data or were assessed to be inaccurate by anatomist project advisors, particularly in the placement of fine detail of nerves and blood vessels. Some products demonstrate a poor relationship between their source data (e.g. MRI) and the modelled structures. Due to our rigourous validation process, the Head and Neck project improves on the accuracy of other anatomical models and has high clinical value, at very fine levels of detail.

- $\quad$ Realism. Several of the anatomical models above are largely diagrammatic, low resolution, and lack definition and detail. The Head and Neck project used laser scanning to capture extremely detailed structural information (e.g. in the surface of the skull) and high resolution digital photography of live patients to ensure realistic textures, at very high levels of definition and detail. The result is colour and structural information that accurately represents the real thing.

- Normalised anatomy. The Head and Neck project is not based on one particular patient but represents a normalised adult male, based on the input and validation of the project's clinical advisory team.

- Functionality/appropriateness to teaching. Whilst many of the products above are highly appropriate for basic medical study, our evaluation showed that many are not of sufficient quality to be useful in a clinical or higher education context. Our Head and Neck model is appropriate for learning, teaching and simulation at an expert level and the functionality supports a wide range of scenarios including: true interactivity (not based on pre-rendered animations); a full suite of interaction tools including 
labelling, zoom, selection, rotation, translation, transparency, guillotine, explode/implode and reassemble; large-scale 3D stereoscopic projection (with optional head-tracking view); control via a PC or game-controller; and an intuitive user interface.

Our systematic review of the medical visualization landscape indicated that there was an unmet need for validated anatomical visualizations of the healthy human body that could be relied upon by viewers as accurate and realistic reproductions of the real thing. Indeed, within medical, dental and surgical curricula, the number of actual contact hours for teaching has markedly reduced over recent years. Within the medical curriculum alone, the General Medical Council issued guidelines to medical schools in the United Kingdom (Tomorrow's Doctors, 1993) requesting a reduction in the amount of factual information [13], [14]. This has happened across many medical schools across the world [13-17]. However, medical training programs also began to change to a more integrated curriculum with various teaching methodologies adopted [18], [19]. More recently in the UK, Tomorrow's Doctors 2009 has placed more emphasis on the medical sciences related to clinical practice. This directly reflects opinion from academics, clinicians and students that the anatomy content had been significantly “dumbed down” previously [17], [20].

Thankfully, this is changing within medical, dental and surgical curricula. Interestingly, with these changes, it has also been shown that to optimise learning, a variety of teaching modalities need to be used alongside traditional techniques. There is now an increased demand from anatomical educators for additional teaching resources, including those of a virtual nature and involving interactive multimedia [21-23]. In addition, the general public as yet has no means to view and interact with a truly representative visual simulation of the human body in a way that provides a genuine educational experience to promote public understanding of health and wellbeing. Using visualization experience gained within the automotive, defence and built environment sectors alongside medical visualization research, we sought to address these shortfalls by constructing a high fidelity 3D dataset, supporting meaningful user engagement, viewing and real-time interaction.

The construction of head and neck anatomy sits very well within the established DDS academic, commercial and research programmes where the primary focus is on user interaction with real-time digital data that supports multi-disciplinary skill sets. It embraces and builds upon our technical development platform for research and commercial development through 3D laser scanning, 2D data capture, data processing and optimisation, 3D construction of objects and environments, photo-realistic rendering, user interface design, real-time display and cross-platform development. There is a direct connection between the development of this anatomical head and neck dataset and our joint MSc in Medical Visualization and Human Anatomy, an innovative programme designed and delivered by the DDS in partnership with the University of Glasgow's Laboratory of Human Anatomy, School of Life Sciences, part of the College of Medical, Veterinary and Life Sciences. Indeed, this collaboration with the Laboratory of Human Anatomy ensures access to one of Europe's largest anatomical facilities with cadaveric material. Continuing collaboration ensures beneficial knowledge exchange and user feedback from this postgraduate student community and continuously informs future research development and in turn positively impacts upon pedagogy and the overall student learning experience.

\section{The Workflow}

Figure 2 shows the development workflow, which at a high level, consists of identification of a suitable donated cadaver, dissection, 3D laser scanning capturing surface measured data, 3D computer modelling of all structures, digital photography from surgical procedures, texture mapping (colour information onto 3D surfaces) and interface development to support user interactions, trials and testing. Verification and validation is conducted at every development stage with final results being presented to a clinical advisory panel who met every three months throughout the project period. 


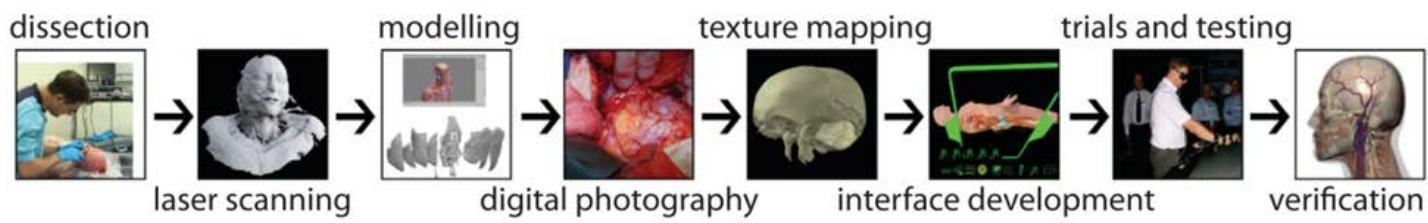

Figure 2. Development workflow

\section{Data Construction}

An important consideration with a project of this size is the sustainability of the workflow, tools and datasets that are generated over its duration. One of the dangers of working within the computing industry is that software and proprietary data formats can often become obsolete over time resulting in data that cannot be read or used. Consequently, it is good practice to ensure that any valuable data is stored using open and welldocumented formats to ensure that it is not reliant on a single company or piece of software to be used. We adopted such an approach for the storage and preservation of the head and neck anatomical datasets. The data generated over the course of this project can be separated into two groups. The first is the raw data such as photographic evidence and point cloud data generated from white light and laser scanners. The second is the processed data created by the modelling team. The non-specialist nature of the raw data enables us to store all of it using open file formats such as the Portable Network Graphics (PNG) format for photographs and images and the Stanford Triangle Format (PLY) for scan data. However, in order to process this data and create the anatomical models, more specialist proprietary tools and data formats are required. Since this cannot be avoided, we used industry standard tools such as Autodesk's Maya and Pixologic's ZBrush to generate and store these models. In order to ensure long-term sustainability, the textured meshes are also exported and stored using an open format such as COLLADA to insure against any of these products becoming obsolete in the future.

\section{Data Acquisition}

We have developed a completely new approach to medical visualization. Our novel data construction workflow and validation process uses donor cadaveric material and through the process of destructive dissection and staged, high resolution laser scanning allows us to produce an accurate 3D anatomical model of head and neck anatomy. To ensure accuracy and a true likeness of the human body, this dataset was validated by the project's Clinical Advisory Board, which comprises anatomists, clinicians and surgical specialists. Our dataset and interface development focuses on real-time interaction and simulation (not pre-rendered animations) that allows users to fully interact with all aspects of the anatomy and can be fully investigated in 3D at any scale, from a laptop or mobile device to a fully immersive environment (see Figure 1).

\section{- Selection of Specimen}

The identification of a suitable embalmed male Caucasian cadaver between the ages of 50-65 was the starting point for this work package. This was identified from the regular stock in the Laboratory of Human Anatomy, School of Life Sciences, College of Medical, Veterinary and Life Sciences at the University of Glasgow. The cadaver had no obvious signs of pre-existing craniofacial abnormalities. All procedures were carried out under the Anatomy Act 1984 [24] and the Human Tissue (Scotland) Act 2006, part 5 [25]. This was undertaken by the government's Licensed Teacher of Anatomy, one of the co-authors (PR). This formed the basis of the definitive 3D digital human that was developed by DDS, with the head and neck region comprising the element to be described. Minimal pre-existing age-related changes were present, significant because the majority of cadavers are of those who have died in old age, many of whom are edentulous. The alveolar bone and teeth were recreated digitally based on laser scans of disarticulated teeth, held at Glasgow Dental School.

- $\quad$ Dissection and data capture of head and neck soft tissue

Ultra high resolution 3D laser scanning supported by high-resolution colour imaging capture was performed on the cadaver before formaldehyde embalming. Perceptron Scanworks V5 3D laser scanner [26] was used to capture accurate data of the surface geometry. Intra-oral scanning was also performed prior to the preservation 
process to allow accurate reconstruction of dental related anatomy (and to establish key anatomical and clinically relevant key landmarks) when there is pliability/mobility at the temporomandibular joint. The embalming procedure was carried out through collaboration of a mortician and qualified embalmer, supervised by a Licensed Teacher of Anatomy in the Laboratory of Human Anatomy, University of Glasgow. The eyes were injected with a saline solution post-embalming to maintain its life-like contour, a technique established in anatomical and surgical training for ocular (and ocular related) surgical procedures designed by the Canniesburn Plastic Surgery Unit, an international leader in plastic and reconstructive training, research and clinical expertise.

Skin and subcutaneous tissue were meticulously dissected (using standard anatomical techniques) from the head and neck territories with appropriate health and safety precautions typical in the environment of using cadaveric tissue. Superficial muscles, nerves, glands and blood vessels were identified. Scanned muscles and attachments included the sternocleidomastoid, infrahyoid muscles, muscles of facial expression (including those around and within the eyes and nose) and the superficial muscles of mastication, including masseter and temporalis, all of which have important clinical and functional applications. The superficial nerves captured at this stage were the major sensory and motor innervations of the head and neck including the trigeminal and facial nerves, and specifically the termination of the facial nerve onto the muscles of facial expression. Scanned glands included the major salivary glands, i.e. the parotid, submandibular and the sublingual glands, as well as the endocrine thyroid gland. The blood vessels identified at this stage are the facial vessels as well as the jugular venous drainage of superficial anatomical structures.

Deeper dissection of the head included data capture for the training of oral related musculature including genioglossus, geniohyoid, mylohyoid, lateral and medial pterygoids, digastric and buccinators amongst others. These specific muscles are significantly important in oral function, and have immense clinical importance for dental training. The related nerve and blood supply to these structures were captured as previously described.

Neck dissection (down to the thoracic inlet) proceeded deeper to identify and capture major and minor structures at this site. Blood vessels (and related branching) were meticulously dissected to demonstrate arterial supply and venous drainage including the common carotid, subclavian and brachiocephalic trunk. Venous drainage includes internal jugular and subclavian veins, and all tributaries. The relationship of these blood vessels to important nerve structures in the neck demonstrated the close proximity to other structures and included the vagus and phrenic nerves, sympathetic trunk and the brachial plexus in the neck (supplying motor and sensory innervation to the upper limbs), and other closely related sensory and motor innervations. Also the larynx and trachea were included in soft tissue structure identification and data capture to clearly show anatomical relations for important clinical procedures, e.g. cricothyroidotomy, tracheostomy and airway intubation, as well as the oesophagus.

Following every stage of identification of all relevant anatomical structures in the head and neck, Perceptron Scanworks V5 laser scanning [26] supported by 3D mesh processing software package, PolyWorks V12 [27] (which aligns the partial scans and generates a mesh surface) were performed prior to the next, deeper dissection. (Figure 3 shows a polygon mesh model generated from raw high-density 3D point clouds). This enabled a complete dataset to be recorded at all stages of dissection, which will then be able to be constructed and deconstructed by users as relevant to the training required, thus creating unique spatial awareness training. 




Figure 3. Surface mesh generated from raw point cloud data

- $\quad$ Scanning of skeletal structures

After soft tissue 3D data capturing, this was removed (apart from ocular structures) to demonstrate the skeletal structures of the head and neck including the neurocranium (calvaria and cranial base), viscerocranium (facial skeleton), mandible and vertebrae. This enables identification of all relevant foramina through which the cranial nerves exit/enter the skull and mandible. Perceptron Scanworks V5 3D laser scanner and PolyWorks were used again to capture the skeletal structures and to process the point cloud data.

Individual laser scans from multiple views of the skull were fused to create a high poly surface mesh, which is the only structure where generated mesh from scan data is directly used in the Head and Neck model. The geometric models of soft-tissue from scan data was found to not accurately represent their natural shape due to soft-tissue deformation resulting from gravity and realignment of the underlying bone structure. Therefore, soft tissue scan data and their mesh are used as a reference in the modelling process and all structures are validated by anatomy specialists to ensure accuracy.

\section{- $\quad$ Intracranial scanning}

At this stage, the vault of the skull and the brain were removed and the same laser scanning and 3D meshing were performed to record specifically all twelve pairs of cranial nerves, cerebral tissue, including parietal, frontal, temporal and occipital lobes with related gyri and sulci, and cerebral vasculature (e.g. Circle of Willis). Where the cranial nerves have been detached, repeat scanning of the base of the skull was undertaken to allow reconstruction of the full intracranial path of these nerves through the numerous foramina to their termination sites. At this stage the visual pathway was established using the gross anatomy of the optic nerves, optic chiasm, optic tracts with modelling of the lateral geniculate body combined with the previous capture of the midbrain and the occipital cortices. Intracranial vascular circulation was modelled based on standard anatomical and applied surgical knowledge.

After the base of the skull was scanned, the roof of the bony orbit was exposed to capture the extra-ocular muscles namely the levator palpebrae superioris, superior, inferior, lateral and medial recti, and the superior and 
inferior oblique incorporating their individual nerve supplies, and related vasculature and nerves surrounding these structures in each of the orbits. The cornea, anterior segment and retina were modelled based on existing anatomical knowledge and understanding.

\section{- $\quad$ Photorealistic Texturing}

At each stage, the capture of 3D topographic data through the use of the Perceptron Scanworks V5 3D laser scanning was supported by high-resolution colour imaging. Since cadavers differ considerably in colour and texture from living tissue, and the shadow, specular highlights, and occlusions in photographs also make them not suitable for texture mapping [28], the photographic data of soft tissue were mainly used as references when building the geometry of models.

In order to produce a photorealistic and accurate model of the aforementioned structures, the skin surface, muscles and skeletal elements consist of several texture layers, describing colour, glossiness and surface structure subtleties. These were achieved through using a combination of photographs of living tissue, the polypainting tool in Zbrush, and various other tools in Photoshop and Autodesk Maya. We produced visually realistic organ textures and appearances that are as close as possible the natural colour of skin and healthy living tissue. Figure 4 shows work-in-progress texturing in Maya.

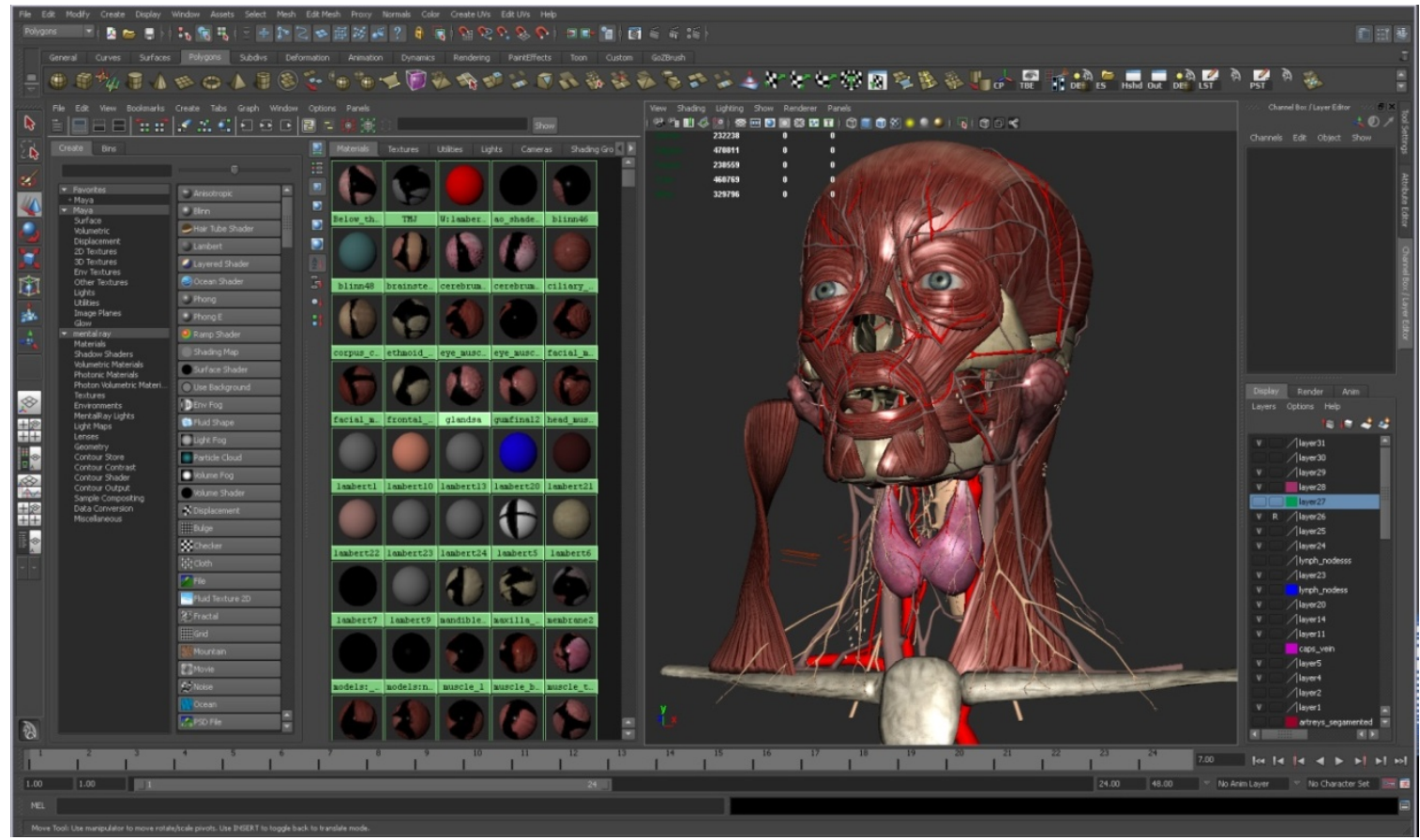

Figure 4. Texturing in Maya

\section{The Complete Dataset of Head and Neck}

The produced high resolution measured dataset is grouped and identified in Maya in order to serve a wide range of anatomical outputs. This enables the interface to present appropriately tagged data to the user, encapsulated in logical subsets. The tagging process includes clinically relevant, anatomically built structures that present context specific information on a case-by-case basis. A rendered head and neck model showing the muscle, nerve, and vascular layers is presented in Figure 5. The resulting complete dataset does not represent any one specific human body but represents a comprehensive structure that captures and presents a normalised, unbiased anatomical model. 




Figure 5. Rendered head and neck showing the muscle, nerve, and vascular layers

\section{Interactive Software}

The uniqueness of the DDS model comes from the forging together of three key components:

- the anatomical precision and accuracy of the constructed datasets;

- $\quad$ the specialist clinical input to challenge and validate the model; and

- $\quad$ the seamless interface allowing proficient user-interactivity in real-time, enabling meaningful feedback and learning.

In order to use the head and neck model in a realtime application, low poly models were created with normal maps baked from a Zbrush sculpt, decimating or re-topologizing to simplify the high poly mesh.

Another importantly unique characteristic is the accompanying suite of manipulation tools, which afford both interactivity (for individual users) as well as interconnectivity (to support collaborative group usage). Figure 6 is a screenshot of the interactive Head and Neck Anatomy, showing a clipping plane on arbitrary angles and available functions in the vertical toolbar on the right. Apart from basic manipulation (such as object translation and rotation) and navigation controls (zoom, pan, etc.), it provides orthographic clipping planes as well as clipping plane on arbitrary angles. The virtual cutting planes reveal cross-section which resembles cryosection or CT imaging.

The users can interact with the Head and Neck either through conventional input methods or through a combination of Xbox controller and head tracking. Together with stereoscopic projection, the latter interface provides an immersive experience for users to explore the internal structure of head and neck. The user can hide and reveal various tissue types, e.g. bones, muscles, nerves, vascular (Figure 6-C), and conduct virtual dissection via a drag-and-drop function (Figure 6-A). An 'explode' mode (Figure 6-B) is available to allow the user to control an explosion via a slider, making the parts separate away from their original locations to reveal the inner details of the head and neck anatomy. Users can also save particular viewpoints and settings which could be loaded in the future (Figure 6-D).

The interactive application also provides data annotation to support teaching and learning. Where clinically relevant, anatomically built structures are appropriately annotated with context specific information on a caseby-case basis to include text related content, linked diagrams and 3D images. 


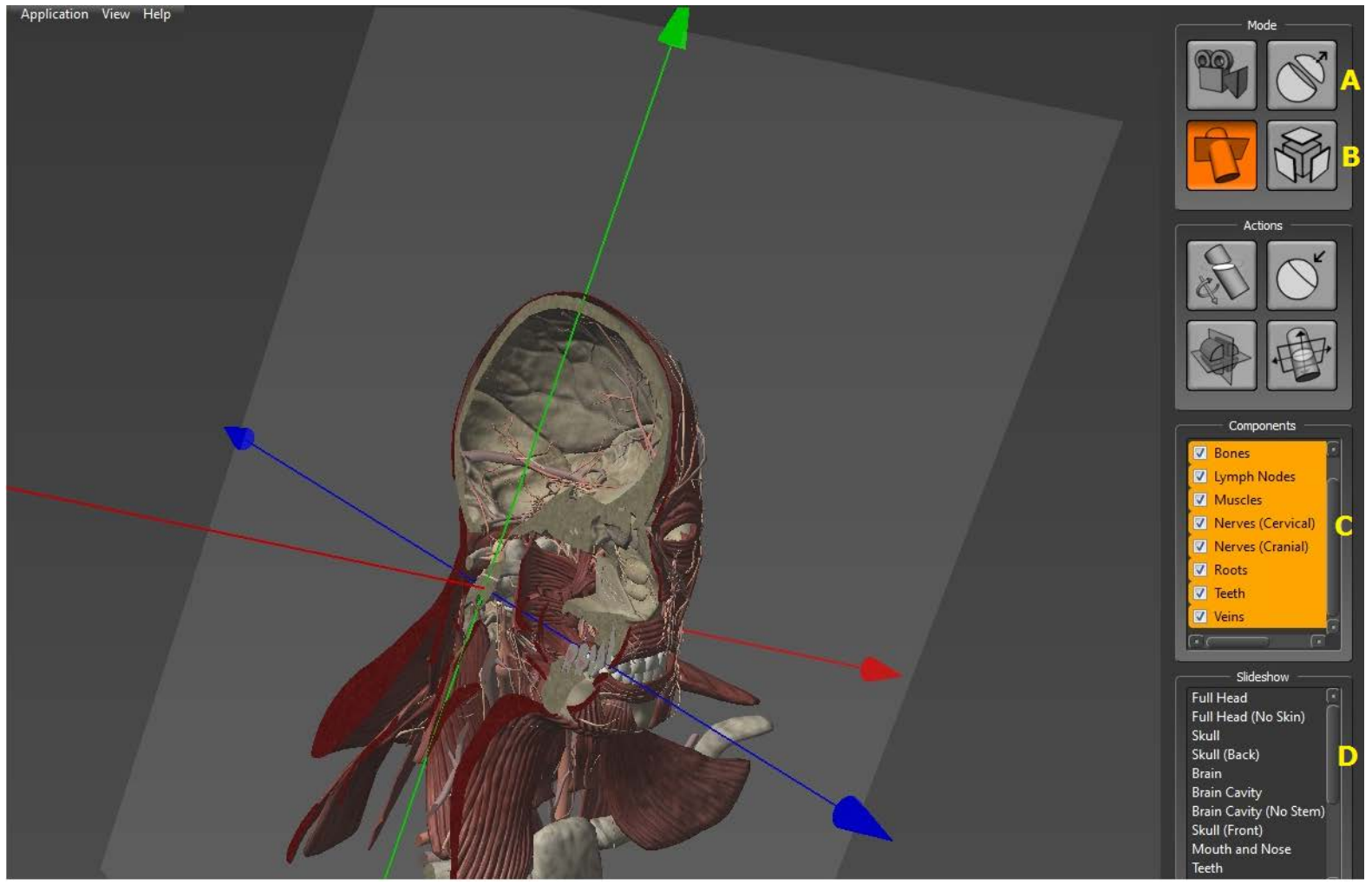

Figure 6. A screenshot of the interactive Head and Neck Anatomy showing clipping plane on arbitrary angles and available features

\section{Verification / Sign off}

Working in collaboration with the 3D modellers in the team, every structure which had been dissected was validated during the modelling process, to ensure anatomical accuracy and a true likeness of the human body. The dataset was validated by the project's Clinical Advisory Board which comprised anatomists, clinicians and surgical specialists. Figure 7 shows an event of clinical validation. PR (one of the co-authors) who is a senior clinical anatomist and government Licensed Teacher of Anatomy, ensured complete anatomical accuracy of all structures constructed in every stage.

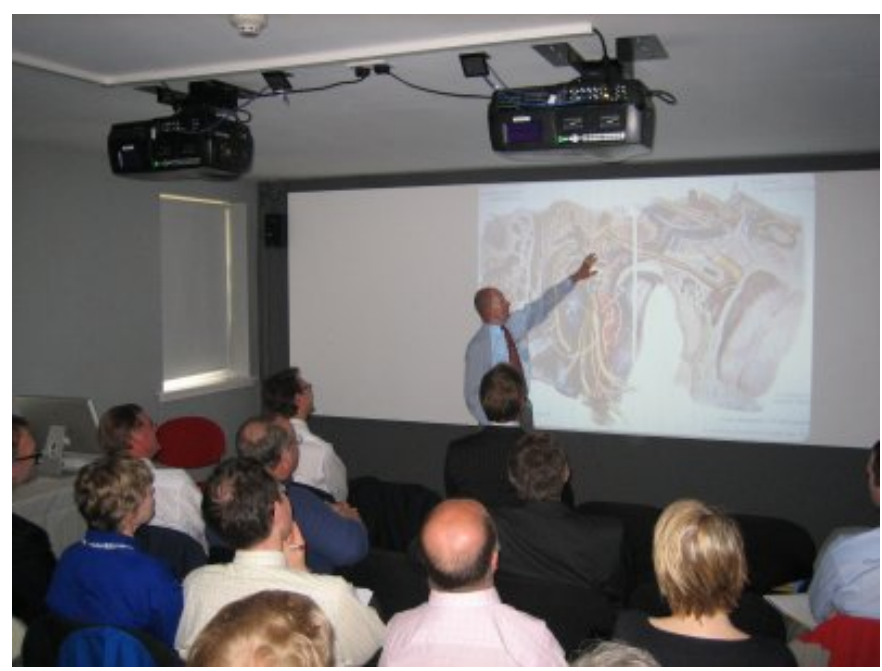

Figure 7. Clinical validation by a clinical advisory board

The skull and mandible were initially reconstructed from the laser-captured skull. Then, a stringent schedule was created and adhered to at the beginning of the project for creating each and every musculoskeletal, vascular, 
neural and glandular structure. The approach undertaken was creating each anatomical structure from the deepest (closest to the skull) to the most superficial i.e. the skin (unlike the superficial to deep dissection). As every muscle, bone, nerve, blood vessel and glandular structure was created it had to be moulded around the framework of the skull. This involved using the laser scanned material and also the high resolution digital photography which was captured for all anatomical structures. This ensured direct correlation with every structure to the dissected, laser-scanned and photographed components.

All attachments, origins, terminations and pathway of every anatomical structure were meticulously created, where the anatomist and digital modellers would assign a set of structures to be modelled during a weekly period over the duration of the project. Therefore, this ensured that a catalogue of ALL anatomical structures in the head and neck was created which had to be modelled from the dissected material. On completion of the modelling of each anatomical structure, this was then reviewed as to its accuracy, initially of that individual element. As the work progressed and the model became more complex, each structure that was created and validated had also to be examined in its accuracy to each and every surrounding anatomical structure to ensure complete rigour and accuracy in position of all structures, including those nearby. This ensured a completely anatomically correct and robust model was being developed. Where relevant, this was also examined by a clinician who operated in that field to ensure not just exceptionally accurate anatomical datasets were being created, but one with all relevant surgical anatomy clearly and accurately identifiable. This was crucial where the anatomy of the oral cavity, including the teeth, had to be created to an exceptionally high level of accuracy never seen to date. This involved a team of oral surgeons and senior dental clinicians working side by side with the modellers during the duration of the project. As each anatomical area (e.g. floor of mouth, intracranial territory, orbit etc) was created in the model, the modellers, anatomist, dental clinicians and surgeons then reviewed those set of structures in that territory and went through a rigorous "signing off" process when the work in that area was complete. To verify the accuracy of the model it was also externally examined and validated by senior academic clinicians specialising in head and neck anatomy. Again, this ensured that each and every anatomical structure gave a real-to-life representation of the structures, as well as ensuring the highest degree of accuracy of the anatomy created.

\section{Haptic Injection}

One of the most commonly performed procedures by a dental practitioner is anaesthetising the inferior alveolar nerve. The development of haptic feedback utilises rigid simulation with great accuracy to enhance student learning. Force feedback devices vary depending on complexity and feedback resolution and have a wide range of downstream applicable scenarios. To this end we use a six-degree of freedom PHANTOM Omni for haptic interaction, as a cost effective solution for larger numbers of users, off-the-shelf console controllers can be utilised in this application. Figure 8 shows the haptic interface for training dental anaesthesia, i.e. an injection which blocks sensation in the inferior alveolar nerve, running from the angle of the mandible down the medial aspect of the mandible, innervating the lower teeth, lower lip, chin, and tongue. The position/orientation and movement of the PHANTOM Omni stylus is linked to a dental syringe. To anesthetize the nerve, the user inserts the needle (stylus) somewhere posterior to the model's last molar. The user can then press one of the PHANTOM Omni pen buttons (Figure 9), which triggers an injection animation, i.e. anaesthetic solution in a breech-loading syringe being injected into soft tissues of the 3D model. The user can feel resistance (force feedback) when soft tissue is touched, and the syringe tip moves smoothly when the injection button is pressed. Visual feedback is also provided through a viewpoint (Figure 8, the third small window on the left of the screen) with the anaesthetic areas turning red. The application also prompts warning messages when, for example, the needle is positioned too posterior and anaesthetic may be put into parotid gland. This application allows dental students unlimited practice opportunities to become familiar with local anaesthetic at zero risk to real patients. 


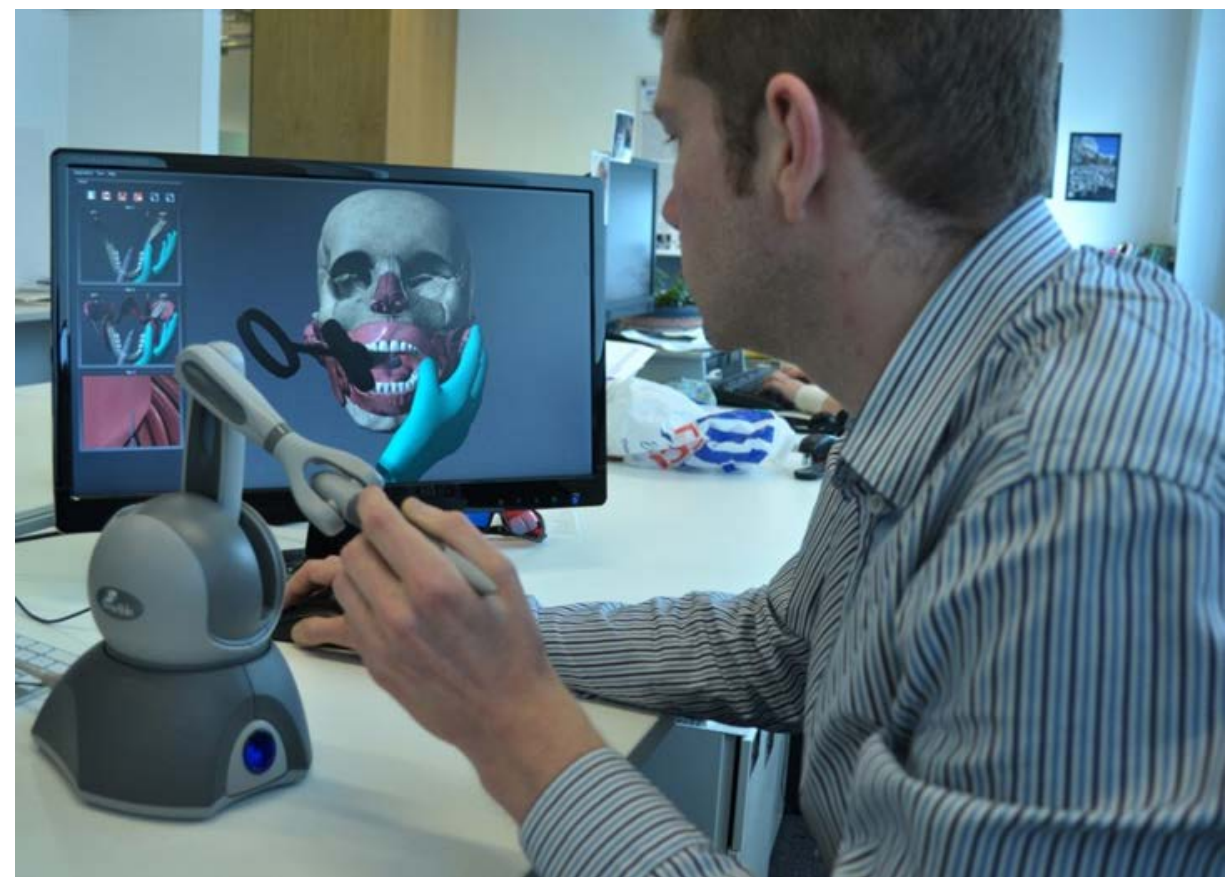

Figure 8. Haptic interaction for training the injection of a local anaesthetic

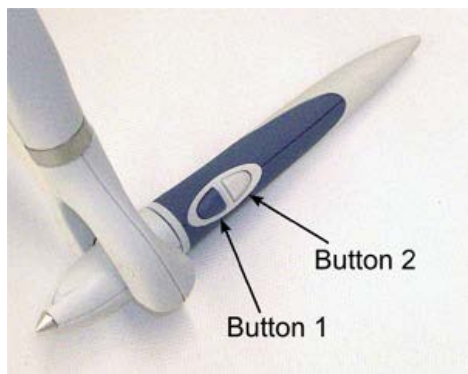

Figure 9. The two PHANTOM Omni pen buttons: one is for injection, the other for reset.

\section{Conclusion and Future Work}

We have described the workflow of data construction, development, and validation of an interactive high resolution three-dimensional anatomical model of head and neck. The Head and Neck datasets represent a step change in anatomical construction, validation, visualization and interaction for viewing, teaching, training and dissemination. In the long term, the results obtained from this three-year project can be viewed as the framework on which to build future efforts. Examples are: to expand models to whole body systems (a project creating a female breast model for breast cancer early detection has already begun); the inclusion of physiological processes (a pilot project on this funded by the Physiological Society has also begun); dynamic representations of the progression of diseases; deformable simulations to model the dynamics of living tissue and physiology, e.g. nerve movement, pulse, blood flow, hemodynamics, collision detection and elasticity and to support surgical rehearsal; and the exploitation of new directions that may include internationalisation initiatives, commercialisation opportunities, and establishment of partnerships with other socially concerned organisations.

\section{References}

[1] Beveridge, E., Ma, M., Rea, P., Bale, K., and Anderson, P. 3D Visualization for Education, Diagnosis and Treatment of Iliotibial Band Syndrome. In the Proceedings of the IEEE International Conference on Computer Medical Applications (ICCMA 2013), Sousse, Tunisia, 20-22 January 2013. ISBN: 978-1-46735213-0, DOI: 10.1109/ICCMA.2013.6506143 
[2] Chang, M.C., Trinh, N.H., Fleming, B.C., and Kimia, B. B. Reliable Fusion of Knee Bone Laser Scans to Establish Ground Truth for Cartilage Thickness Measurement. In SPIE Medical Imaging (Image Processing, Proceedings of SPIE Volume 7623). San Diego, CA. Feb. 2010.

[3] Kalea, E.H., Mumcuoglua, E.U., Hamcanb, S. Automatic segmentation of human facial tissue by MRI-CT fusion: A feasibility study. Computer Methods and Programs in Biomedicine, 108(3):1106-1120, December 2012. Elsevier.

[4] Anderson, P. Developing 3D Interactive Anatomy. The RCPSG MacEwen lecture at the International Surgical Congress of the Association of Surgeons of Great Britain and Ireland, Glasgow, 2009.

[5] RCUK and Universities UK. Big Ideas for the Future. June 2012. [accessed 13 May 2013] Available from: http://www.rcuk.ac.uk/Publications/reports/Pages/BigIdeas.aspx

[6] VisibleBody [accessed the 18 May 2013] Available from: http://www.visiblebody.com

[7] Body Browser [accessed the 18 May 2013] Available from: http://www.zygotebody.com/

[8] Primal Pictures [accessed the 18 May 2013] Available from: http://www.primalpictures.com/

[9] Cyber Anatomy [accessed the 18 May 2013] Available from: http://cyber-anatomy.com

[10] Spitzer, V., Ackerman, A., Scherzinger, A., \& Whitlock, D. (1996). The Visible Human Male: A Technical Report. Journal of American Medical Informatics Association, 3 (2), 118-130.

[11] 3D4Medical [accessed the 18 May 2013] Available from: http://www.3d4medical.com

[12] BodyViz [accessed the 18 May 2013] Available from: http://www.bodyviz.com/

[13] Utting, M., Willan P. (1995). What future for dissection in courses of human topographical anatomy in universities in the UK. Clin Anat 8:414-417.

[14] Dangerfield, P, Bradley P, Gibbs T. (2000). Learning gross anatomy in a clinical skills course. Clin Anat 13:444-447.

[15] Collins, T.J, Given, R.L., Hulsebosch, C.E., Miller, B.T. (1994). Status of gross anatomy education as perceived by certain postgraduate residency programs and anatomy course directors. Clin Anat 7:275-296.

[16] Holla, S.J., Selvaraj, KG, Isaac, B, Chandi, G. (1999). Significance of the role of self-study and group discussion. Clin Anat 15:38-44.

[17] Fitzgerald, J.E., White, M.J., Tang, S.W., Maxwell-Armstrong, C.A., James, D.K. (2008). Are we teaching sufficient anatomy at medical school? The opinions of newly qualified doctors. Clin Anat 21:718-724.

[18] Schmidt, H. (1998). Integrating the teaching of basic sciences, clinical biopsychosocial issues. Acad Med 73:S24-S31.

[19] Ling Y, Swanson DB, Holtzman K, Bucak SD. (2008). Retention of basic science information by senior medical students. Academic Medicine, 83:S82-85.

[20] Patel, K.M., Moxham, B.J. (2006). Attitudes of professional anatomists to curricular change. Clin Anat 19:132-141.

[21] Kluchova, D., Bridger, J., Parkin, I.G. (2000) Anatomy into the future. Bratisl Lek Listy. 101(11):626-629

[22] Turney, B.W. (2007) Anatomy in a modern medical curriculum. Ann R Coll Surg Engl. 89:104-107

[23] Sugand K, Abrahams, P. and Khurana, A. The anatomy of anatomy: A review for its modernization. Anatomical Sciences Education 3(2):83-93, 2010.

[24] Anatomy Act 1983, [accessed the 15 May 2013] Available from: http://www.legislation.gov.uk/ukpga/ 1984/14/pdfs/ukpga_19840014_en.pdf

[25] Human Tissue (Scotland) Act 2006. [accessed on the $15^{\text {th }}$ May] Available from: http://www.legislation.gov. uk/asp/2006/4/pdfs/asp_20060004_en.pdf

[26] Perceptron Scanworks V5. [accessed the 16 May 2013] Available from: http://www.exactmetrology.com/ products/perceptron/scanworks-v4i/

[27] PolyWorks. [accessed the 16 May 2013] Available from: http://www.innovmetric.com/polyworks/3Dscanners/home.aspx

[28] Cenydd, L., John, N.W., Bloj, M., Walter, A., and Phillips, N.I. Visualizing the Surface of a Living Human Brain. IEEE Computer Graphics and Applications, March/April 2012, 55-65. 